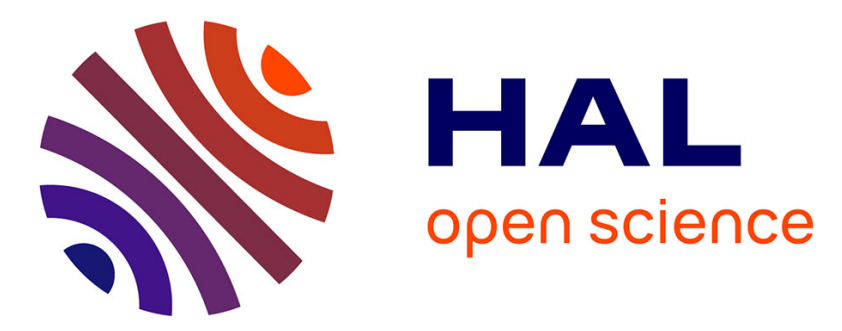

\title{
Resistance and 1/f noise between circular contacts on conductive thin films
}

\author{
C. Liang, L. K.J. Vandamme, G. Leroy, J. Gest
}

\section{To cite this version:}

C. Liang, L. K.J. Vandamme, G. Leroy, J. Gest. Resistance and 1/f noise between circular contacts on conductive thin films. European Physical Journal: Applied Physics, 2011, 53 (3), 10.1051/epjap/2010100404 . hal-00672780

\section{HAL Id: hal-00672780 \\ https://hal.science/hal-00672780}

Submitted on 22 Feb 2012

HAL is a multi-disciplinary open access archive for the deposit and dissemination of scientific research documents, whether they are published or not. The documents may come from teaching and research institutions in France or abroad, or from public or private research centers.
L'archive ouverte pluridisciplinaire HAL, est destinée au dépôt et à la diffusion de documents scientifiques de niveau recherche, publiés ou non, émanant des établissements d'enseignement et de recherche français ou étrangers, des laboratoires publics ou privés. 


\section{Resistance and $1 / f$ noise between circular contacts on}

\section{conductive thin films}

C. Liang ${ }^{a b}$, L. K. J. Vandamme ${ }^{c}$, G. Leroy ${ }^{a b}$, and J. Gest ${ }^{a b}$

${ }^{a}$ Université Lille Nord de France, 5900 Lille, France

${ }^{\mathrm{b}}$ Unité de Dynamique et Structure des Matériaux Moléculaires, Université du Littoral

Côte d'Opale, 50 rue F. Buisson, 62228 Calais, France

${ }^{\mathrm{c}}$ Eindhoven University of Technology, Department of Electrical Engineering PT 913

P.O. Box 513, 5600MB Eindhoven, The Netherlands 


\begin{abstract}
There is a need to characterize the quality of contacts and the noise properties of new materials deposited or grown as thin films. Poor contacts are interface dominated. Perfect contacts have a negligible interface contribution and there is only a resistance and noise contribution from outside the contact region. The presence of current crowding enhances the resistance and noise contribution. Such contacts are called constriction dominated contacts. The conductive film is characterized by its sheet resistance and normalized conductance fluctuations for a unit surface. The resistance and noise is studied between two circular top electrodes of the same diameter on the conductive. To distinguish between perfect and poor contacts and to characterize the thin film in case of good contacts, we need a set of contacts with different diameters. Models for perfect and poor contacts are investigated. The scaling of resistance and noise with contact radius $r$ is for interface dominated poor contacts: $R_{i} \propto 1 / r^{2}$ and $S_{R_{i}}$ $\propto 1 / r^{6}$. In contrast, perfect contacts with contact diameter $(2 r)$ much smaller than the distance between the centers $(2 b)$ show: $R_{c} \propto \ln (b / r)$ and $S_{R c} \propto 1 / r^{2}$. From the resistance and noise measurements between constriction dominated perfect contacts, the sheet resistance and normalized noise of the thin film are calculated.
\end{abstract}

Keywords: thin film, contact resistance, $1 / f$ noise

PACS : 7270+m, 7340cg, 7440+k 


\section{Introduction}

The preparation of perfect contacts on new materials deposited or grown as thin layers remains a challenge [1]. There is a need to test the quality of the contacts. In the standard method a set of parallel line shaped contacts is used at different distances on a rectangular sample. This is the so-called transmission line structure for contact resistivity measurements (conventional TLM and improved) [2]. The Cox and Strack geometry for example, involves fabrication of circular contacts of variable radii on the top side of the material and a blanket contact on the bottom [3].

Here, we present a test method for contacts on thin films using resistance and noise measurements between a pair of circular top electrodes of variable radius. From the different dependence between resistance and radius for poor and perfect contacts we can distinguish between interface dominated and constriction dominated contacts. The difference in dependence on radius for the noise in the resistance is even more outspoken.

The resistance and $1 / f$ noise for perfect circular contacts was calculated and compared with experimental results in Ref. [4]. In this case the sheet resistance $R_{s h}$ and normalized noise for a unit surface $C_{u s}$ of the thin film can be characterized. The $R_{s h}$ and $C_{u s}$ are easily defined for a rectangular sample of width $W$ and distance $L$ between two perfect line contacts as follows:

$$
R=R_{s h} \frac{L}{W} \Rightarrow R_{s h}=R \frac{W}{L}[\Omega] \Leftrightarrow \frac{S_{R}}{R^{2}}=\frac{\alpha}{N f}=\frac{\alpha}{n_{2} W L f}=\frac{C_{u s}}{W L f} \Rightarrow C_{u s}=\frac{f S_{R}}{R^{2}} W L\left[m^{2}\right]
$$

The relative noise is inversely proportional to the number of free carriers $N$ between the line contacts: $N=n_{2} W L$ where $n_{2}$ is the two-dimensional free carrier concentration and $\alpha$ is the $1 / f$ noise parameter [5-7]. 
Here, we present two approximations for the calculations on small perfect contacts: the first one is based on the limit of the exact results in Ref. [4], and the second one is based on an approximation of the equipotentials by concentric circles around the contacts.

In a third approximation the interface dominated contacts are studied as in Ref. [6]. The approximations result in simple relations between contact radius, resistance and noise for perfect and poor contacts. The dependence on diameter is quite different for constriction and interface dominated contacts. This is used to distinguish between perfect and poor contacts as in Ref. [4, 5].

Here, the test method is used to characterize polyaniline / polyurethane conductive polymers prepared with circular golden contacts. The results are compared with four point measurements [8].

\section{Model for constriction dominated contacts (perfect contacts)}

Here, we assume no contribution of the interface. The contact configuration is shown in Fig. 1(a). The approximation for the equipotential lines as concentric circles is shown in Fig. 1(b).

The calculation for the resistance is based on the following integral:

$$
R_{c}=\frac{1}{I^{2}} \int_{A} R_{s h} J^{2} d A
$$

where $I$ is the applied current, $R_{s h}$ is the sheet resistance also called the two dimensional resistivity with dimension $[\Omega]$ and $J$ is the two dimensional current density with dimension $[\mathrm{A} / \mathrm{m}]$ and $d A$ an elementary area. For a rectangular sample of width $W, J=I / W$ and the resistance using Eq. (1) is $R=R_{s h} L / W$ as expected. 
From Eq. (1) and the relation for $J$ between circular contacts follows [4]:

$$
R_{c}=\frac{R_{s h}}{\pi} \ln \left[\frac{b}{r}+\sqrt{\left(\frac{b}{r}\right)^{2}-1}\right]
$$

The relation for $J$ in Ref. [4] is a good approximation for circular contacts $2 b$ apart and placed in the middle of a film with dimensions of at least $10 b \times 10 b$. For small contact radii $b / r>>1$, we find an approximation for Eq. (2)

$$
R_{c}=\frac{R_{s h}}{\pi} \ln \left(2 \frac{b}{r}\right)
$$

For $b / r>>1$ an approximation for the current density can be used, which is based on concentric circular equipotentials. Then the current density is $J=I /(2 \pi x)$ for $r<x<b$ as in Fig. 1b. With Eq. (1) the approximated resistance can be written as:

$$
R_{c}=2 \frac{R_{s h}}{I^{2}} \int_{r}^{b}\left(\frac{I}{2 \pi x}\right)^{2} 2 \pi x d x=\frac{R_{s h}}{\pi} \ln \frac{b}{r}
$$

The exact and approximated expressions for $R_{c}$ in Eq. (2-4) are shown in Fig. 2(a).

The $1 / f$ resistance noise is given by Ref. $[4,6]$

$$
S_{R}=\frac{R_{s h}^{2} C_{u s}}{f \quad I^{4}} \int_{A} J^{4} d A
$$

where $C_{u s}=\alpha / n_{2}$ with $\alpha$ the $1 / f$ noise parameter [7] and $n_{2}$ is the two-dimensional free carrier concentration, $n_{2}=n \times t$ with $n$ the three-dimensional concentration and $t$ the thickness of the layer. $C_{u s}$ is the so-called normalized resistance noise for twodimensional conductors. For a simple rectangular sample of width $W$ and length $L$ between two line contacts holds $J=I / W$ and with Eq. (5) holds 
$S_{R}=\frac{R_{s h}^{2} C_{u s}}{f} \frac{L}{W^{3}}=\frac{C_{u s}}{f W L} R^{2}$

Using the exact expression for current density $J$ in Eq. (5) the $1 / f$ resistance noise between two circular contacts of the same diameter is [4]

$S_{R_{c}}=\frac{R_{s h}^{2} C_{u s}}{4 \pi^{3} f} \frac{1}{r^{2}} \frac{(b / 2 r) \sqrt{(b / r)^{2}-1}+\ln \left[b / r+\sqrt{(b / r)^{2}-1}\right]}{(b / r)^{2}-1}$

For large values of $b / r$ holds

$S_{R_{c}}=\frac{R_{s h}^{2} C_{u s}}{8 \pi^{3} f} \frac{(b / r)^{2}+2 \ln (2 b / r)}{b^{2}}$

If the approximation of the current density between concentric circular equipotentials is used as in Fig. 1(b) we find with Eq. (5)

$S_{R_{c}}=2 \frac{R_{s h}^{2} C_{u s}}{f} \frac{1}{I^{4}} \int_{r}^{b}\left(\frac{I}{2 \pi x}\right)^{4} 2 \pi x d x=\frac{R_{s h}^{2} C_{u s}}{f 8 \pi^{3}}\left[\frac{1}{r^{2}}-\frac{1}{b^{2}}\right]=\frac{R_{s h}^{2} C_{u s}}{8 \pi^{3} f} \frac{(b / r)^{2}-1}{b^{2}}$

Hence, smaller contact radii result in a higher resistance and higher noise with $S_{R c} \propto(b / r)^{2}$ for $b / r>1$.

The exact and approximated expressions for $S_{R c}$ in Eq. (7-9) are shown in Fig. 2(b).

\section{Model for interface dominated contacts (poor contacts)}

Here, we assume only the dominant contribution from an interface of unknown thickness $t_{i}$ and unknown resistivity $\rho_{i}$ and dominant noise contribution between the metal contact and the film. Hence,

$R_{i}=2 \frac{\rho_{i} t_{i}}{\pi r^{2}}=2 \frac{\rho_{\text {cont }}}{\pi r^{2}}$ 
where $\rho_{\text {cont }}=\rho_{i} t_{i},\left[\Omega \mathrm{cm}^{2}\right]$

We assume that even for the largest radii holds: $R_{i}>>R_{c}$ or $\frac{\rho_{\text {cont }}}{r^{2}}>R_{s h}$.

For the noise holds [5]

$S_{R_{i}}=\frac{\alpha_{i}}{N_{i} f} R_{i}^{2}=\frac{\alpha_{i}}{2 n_{i} \pi r^{2} t_{i} f}\left[\frac{2 \rho_{\text {cont }}}{\pi r^{2}}\right]^{2}$ with $C_{u s_{i}} \equiv \frac{\alpha_{i}}{n_{i} t_{i}} \Rightarrow S_{R_{i}}=\frac{C_{u s_{i}}}{f}\left[\frac{2 \rho_{\text {cont }}{ }^{2}}{\pi^{3} r^{6}}\right]$

where $N_{i}$ is the number of carriers in the interface, $n_{i}$ the concentration of free carriers in the interface, $\alpha_{i}$ the noise parameter for the interface, and $\pi r^{2} t_{i}$ the volume of the interface.

For poor contacts (interface dominated) we assume that the normalized noise of the interface $C_{u s i}>C_{u s}$ of the thin film.

The trends in the dependence on contact radius for perfect and poor contacts are summarized in Table 1 and Fig 3. In Fig. 3 the resistance and noise of perfect and poor contacts is shown in normalized and dimensionless values. The resistance of perfect contacts is calculated from Eq. (4) in a normalized resistance $R_{c \text { norm }}$ defined as $R_{\text {c norm }} \equiv R_{c} \frac{\pi}{R_{s h}}=\ln \frac{b}{r}$

The normalized noise $S_{R c \text { norm }}$ is calculated with Eq. (9) as

$$
S_{R_{c \text { norm }}} \equiv S_{R_{c}} \frac{8 \pi^{3} b^{2} f}{R_{s h}^{2} C_{u s}}=\left(\frac{b}{r}\right)^{2}-1
$$

The poor contact is from Eq. (10) and calculated in normalized resistance $R_{\text {inorm }}$ as

$$
R_{\text {inorm }} \equiv R_{i} \frac{\pi b^{2}}{2 \rho_{\text {cont }}}=\left(\frac{b}{r}\right)^{2}
$$


The noise in Fig. 3 is from Eq. (11) and calculated in normalized values $S_{R_{i} \text { norm }}$ as

$$
S_{R_{\text {inorm }}} \equiv S_{R_{i}} \frac{\pi^{3} b^{6} f}{2 C_{\text {us } i} \rho_{\text {cont }}^{2}}=\left(\frac{b}{r}\right)^{6}
$$

\section{Measurement setup}

The noise was measured from a Hewlett Packard HP 89410A Vector Signal Analyzer. The sample was biased by batteries powered voltage source through a large resistance connected in series in order to reveal the level of $1 / f$ noise. All resistors were wirewound to reduce excess $1 / f$ noise. The voltage signal delivered by the sample was amplified by an EG\&G 5184 low noise voltage amplifier, also powered by batteries, before being applied to the input of the analyzer. The sample, voltage source, amplifier and resistor were placed in a shield box during measurement. The data were captured and downloaded to a computer for data storage, display and analysis. An example of measured spectra and a schematic view of the completed device and a block diagram of the electrical circuit used for low frequency noise measurement are shown in Fig. 4. The frequency ranges of our set-up were $0.1 \mathrm{~Hz}-1 \mathrm{MHz}$.

\section{EXPERIMENTAL RESULTS}

The experiments were carried out on a conductive polymer layer, using two circular golden contacts, deposited through a mask by evaporation. After measuring the resistance and the noise, a new pair of contacts with a larger diameter was deposited over the existing contacts. The chosen current bias and frequency is so that the $1 / \mathrm{f}$ noise is always higher than the thermal noise and background noise. The results are recorded in Table 2 . The distance between 2 contact centers is $20 \mathrm{~mm}$. We started the measurements by injecting a constant current between a pair of contacts of $1.5 \mathrm{~mm}$ in 
diameter, the current level $I$ did not exceed $5 \mathrm{~mA}$. Then the diameter of contacts increased, the distance between two circular contacts decreased, we needed a stronger current level. For contacts of $8 \mathrm{~mm}$ in diameter, the maximum current level $I$ was smaller than $50 \mathrm{~mA}$. From the observed voltage noise $S_{V}$ across the circular contacts, the resistance noise is calculated as $S_{R}=S_{V} / I^{2}$. The observed low-frequency noise has a spectrum proportional to $1 / f^{\gamma}$ with the exponent $\gamma$ in the range 0.95 to 1.05 . This is generally called $1 / f$ noise. We used the best $1 / f$ fit through the experimental results to calculate the frequency independent value, $f S_{R}(f)$ with dimension $\left[\Omega^{2}\right]$. This value corresponds with the value at $1 \mathrm{~Hz}$ of $S_{R}(f)$ with dimension $\left[\Omega^{2} / \mathrm{Hz}\right]$.

For contacts of $8 \mathrm{~mm}$ in diameter the observed $S_{V}$ was of the same order of magnitude as the background noise without current through the sample. For this reason the noise result of the $8 \mathrm{~mm}$ diameter is not presented and diameters larger than $8 \mathrm{~mm}$ were not deposited. The measured resistance is quite close to the curve shown in Fig. (5) which is calculated with Eq. (2) by using $R_{s h}=11.5 \Omega$. This shows that the interface contribution to the contact resistance can be ignored.

The noise always depends on contact geometry and on a noise source factor $C_{u s}$ (for the film of a unit surface) as can be seen in Eqs. (6-9). The normalized noise $C_{u s}=$ $1.3 \times 10^{-10} \mathrm{~mm}^{2}$ of this conductive polymer was obtained by a four point measurements [8]. Using in Eq. (7) $C_{u s}$ and $R_{s h}$ found previously, we have calculated the curve of $S_{R}$ versus $b / r$ (see Fig. 5), the measurement results obtained between two circular contacts are indicated by squares. The results scatter around the calculated line. It is well known that resistance noise is much more sensitive for in homogeneities in the material than the resistance itself as was discussed in Ref. $[9,10]$. The fact that resistance and resistance noise follow the calculated lines for constriction dominated 
(perfect) contacts is an indication for the good quality of the contacts. This means that our contacts are not dominated by interface contributions, neither for the resistance nor for the noise. Nevertheless, the estimated upper limit for the interface contribution from the contact with smallest diameter is $\rho_{\text {cont }}<2 \Omega \mathrm{cm}^{2}$. Values as high as $2.5 \Omega \mathrm{cm}^{2}$ have been observed on $\mathrm{CuS}$ layers in literature [5].

In addition, interface dominated contacts have in general the normal thermal noise and a strong $1 / f$ noise. In some cases interface noise can have a generation recombination type noise leading to the typical Lorentzian spectra like in Ref. [11]. The validity of the theoretical results for interface noise with a generation recombination spectrum still holds, because the scaling of interface noise with the diameter is the same for $1 / f$ noise and generation recombination noise.

\section{CONCLUSION}

In this study we have calculated and compared the resistance and normalized noise of a thin film with perfect circular contacts: (i) the general case for any value of $b / r$; (ii) the case with small contact radii (large value for $b / r$ ); (iii) the approximation of the circular equipotential lines.

In addition we have calculated the resistance and noise for poor (interface dominated) contacts: $R_{i} \propto 1 / r^{2}$ and $S_{R_{i}} \propto 1 / r^{6}$.

The experimental results on golden contacts deposited on polymer layers show that the resistance and the noise are not influenced by contact interface contributions. The typical proportionalities $R \propto \ln (b / r)$ and $S_{R c} \propto 1 / r^{2}$ for perfect contacts are observed with the correct value for the proportionality factors $R_{s h}$ and $C_{u s}$ as obtained from independent four point measurements. 


\section{References}

[1] L.J. Brillson, Contacts to semiconductors Fundamentals and Technology, Park

Ridge, New Jersey, USA: Noyes Publications, (1993)

[2] L.P. Floyd, T. Scheuermann, P.A.F. Herbert and W.M. Kelly, Solid-State Electronics, 37, p. 1579-1584, (1994)

[3] M. Ahmad, T. Ganguli, S. Patil, S. Major and Y.G.K. Patro, Solid-State Electronics, 38, p. 1437-1440, (1995)

[4] L.K.J. Vandamme and J.C.F. Groot, Electronics Letters, 14, p. 30-32, (1978)

[5] L.K.J. Vandamme and A. Douib, Solid-State Electronics, 25, p. 1125-1127, (1982)

[6] L.K.J. Vandamme and A.H. de Kuijper, Solid-State Electronics, 22, p. 981986, (1979)

[7] F.N. Hooge, T.G.M. Kleinpenning and L.K.J. Vandamme, Reports on Progress in Physics, 44, p. 479-532, (1981)

[8] C. Liang, G. Leroy, J. Gest, L.K.J. Vandamme and J-L. Wojkiewicz, Synthetic metals, $159, \mathrm{n}^{\circ} 1-2$, p. 1-6, (2009)

[9] L. K. J. Vandamme, IEEE Transactions on electron devices, 41, $\mathrm{n}^{\circ} 11,: \mathrm{p}$. 2176-2187, (1994)

[10] L. K. J. Vandamme and G. Trefan. IEE Proceedings - Circuits devices and systems, $149, \mathrm{n}^{\circ} 1$, p. 3-12, (2002)

[11] E. P. Vandamme and L. K. J. Vandamme. Diagnostics of the quality of MOSFETs. Microelectronics reliability, 36, n 7-8, p. 1107-1112, (1996) 
Figure captions

Fig. 1. (a). Circular contacts of diameter $2 r$ at a distance $2 b$ apart. (b). The approximated concentric circular equipotentials at a distance $x$ from the contact centre.

Fig. 2. (a). Calculated resistance of constriction dominated contacts (perfect contacts) as a function of the geometry: upper, middle and lower lines respectively represent the approximated expression for small $r$ (Eq. (3)), the exact expression (Eq. (2)), and the approximated expression based on circular equipotentials (Eq. (4)).

Fig. 2. (b). Calculated contact resistance noise of constriction dominated contacts (perfect contacts) as a function of the geometry: upper, middle and lower lines respectively represent the exact expression (Eq. (7)), the approximated expression for small $r$ (Eq. (8)), and the approximated expression based on circular equipotentials (Eq. (9)).

Fig. 3. Comparison between perfect (constriction dominated) and poor (interface dominated) contacts in normalized values versus $b / r$ using Eq. (12-15).

Fig. 4. Example of measured spectra, device structure and electrical circuit used for low frequency noise measurement for sample film. 
Fig. 5. Comparison between the experimental plots and the calculated plots based on perfect contacts assumption. 


\section{Table captions}

Table 1. Trends between resistance and noise and contact diameter

Table 2. Resistance and noise results on conducting polymers 


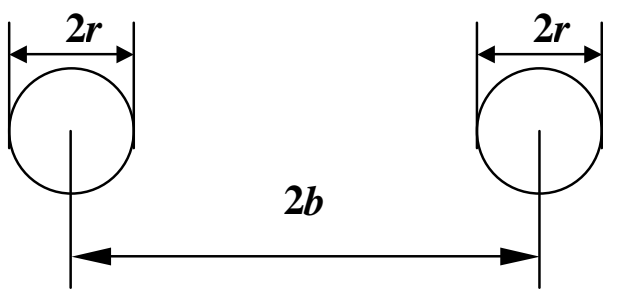

(a)

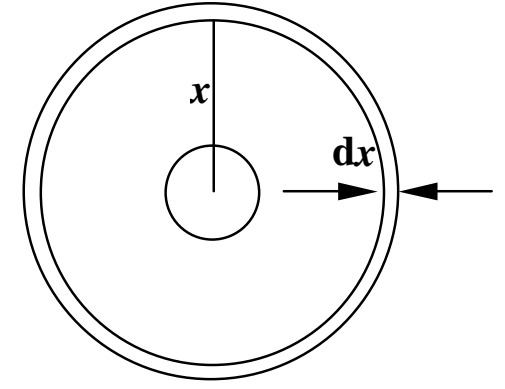

(b)

Fig. 1.

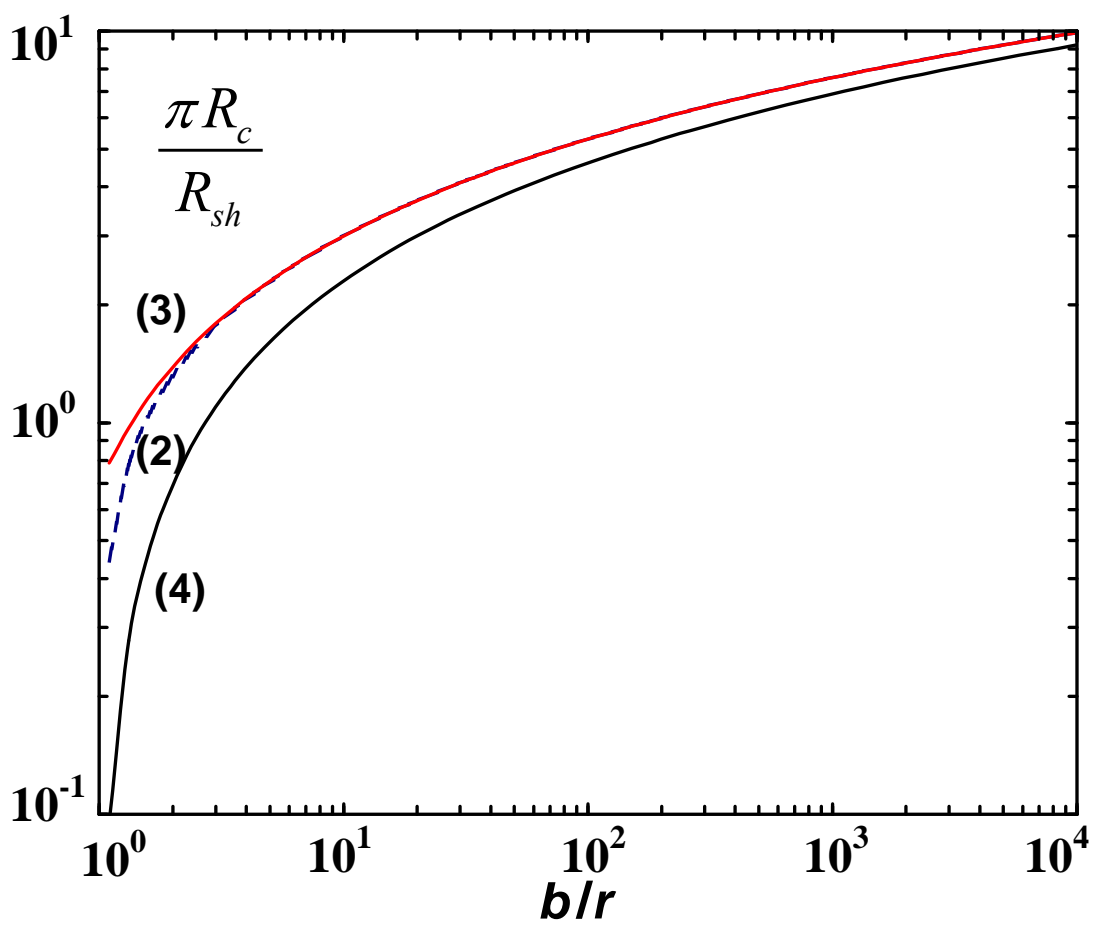

Fig. 2. (a). 


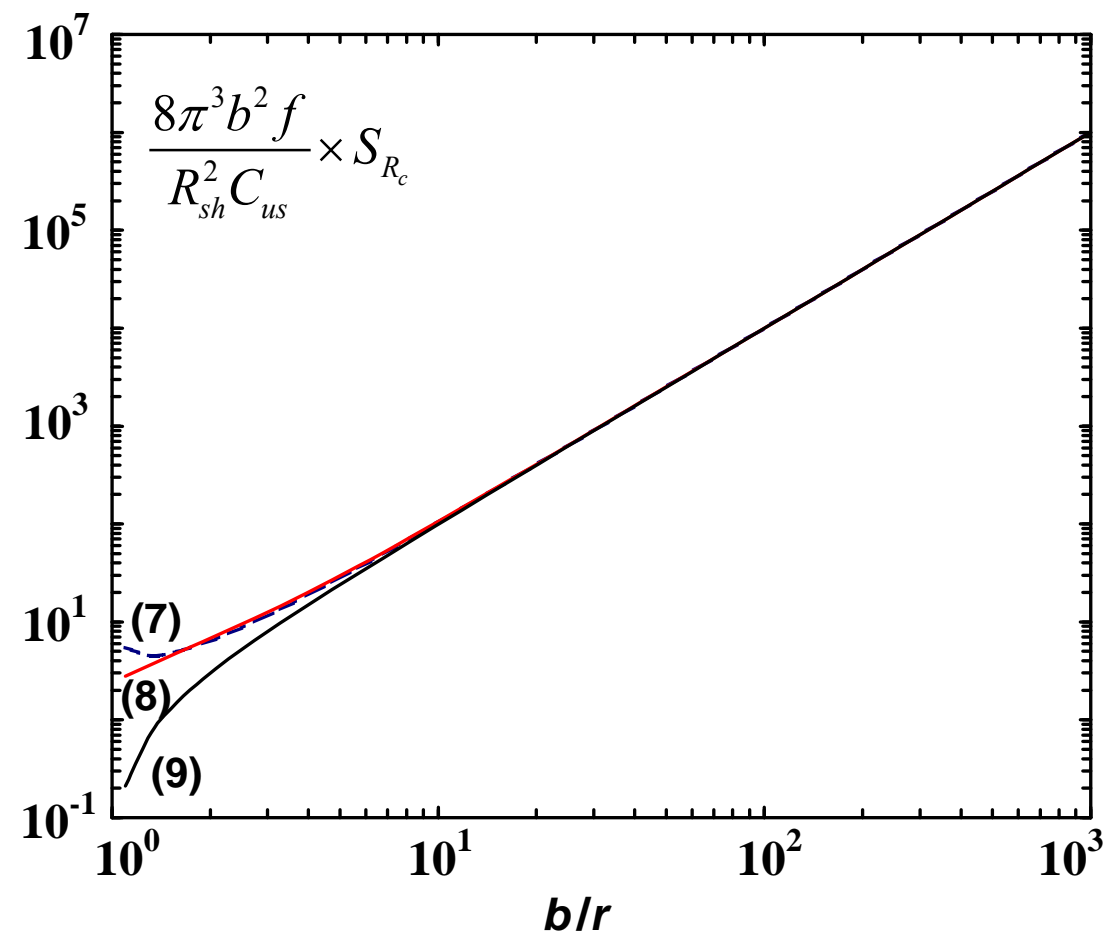

Fig. 2. (b).

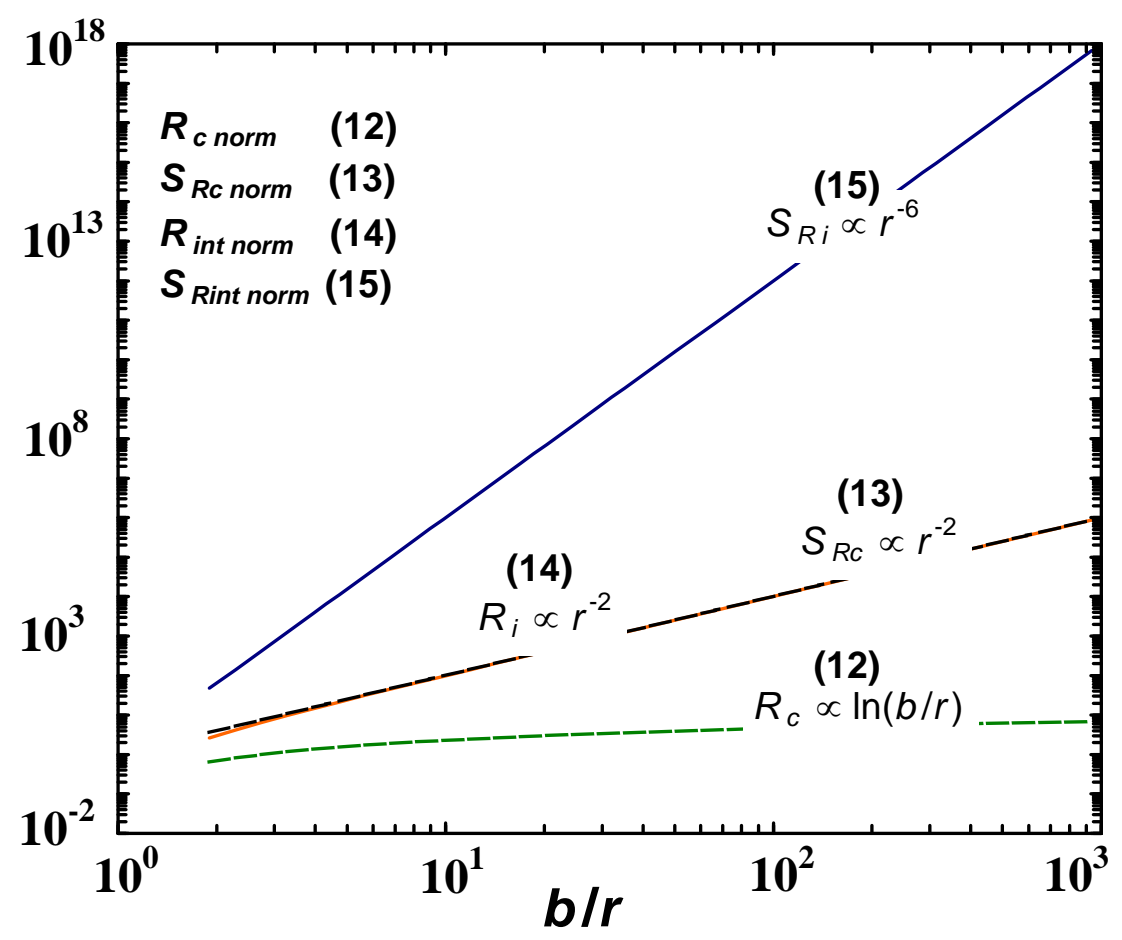

Fig. 3. 


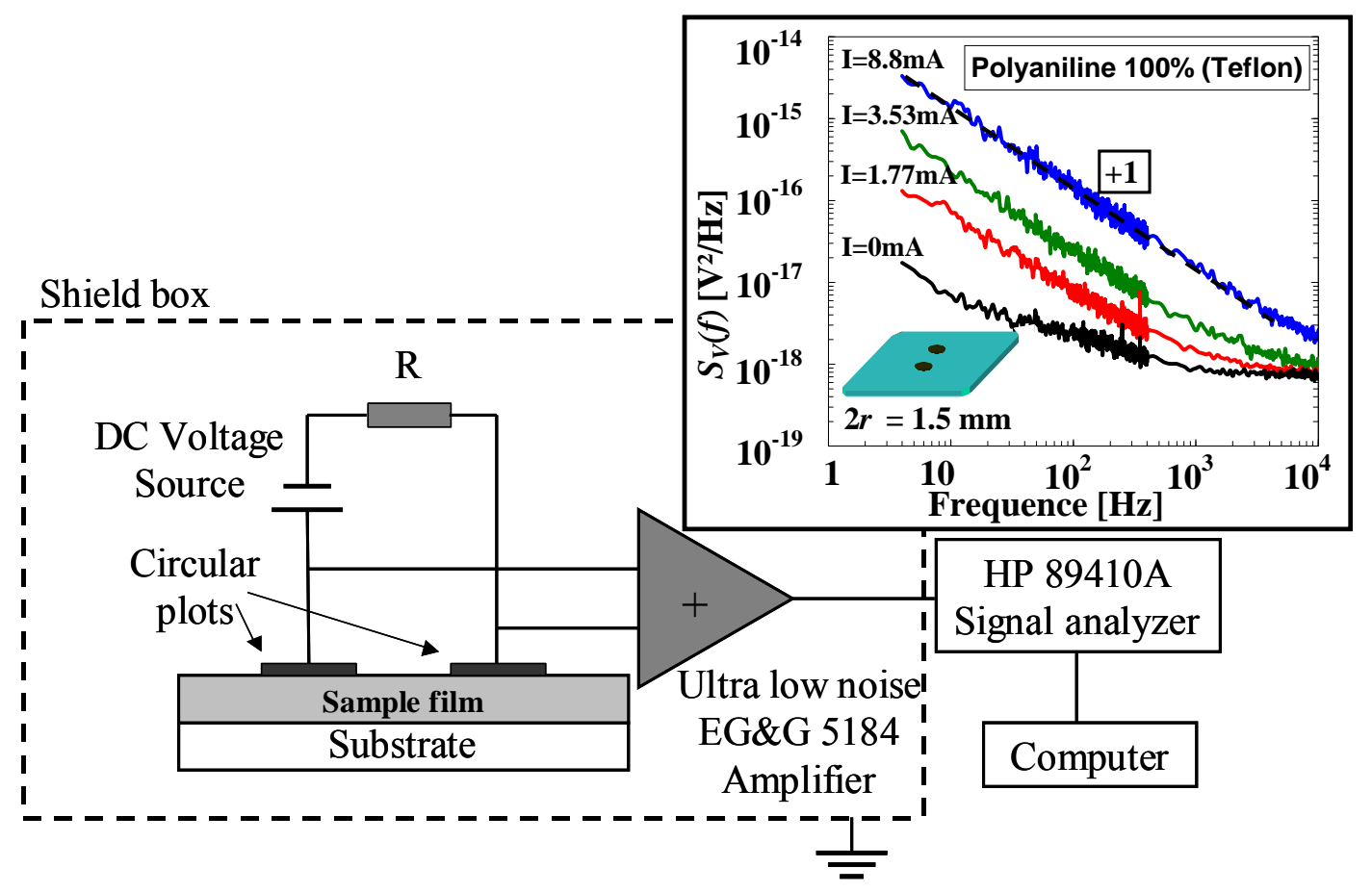

Fig. 4.

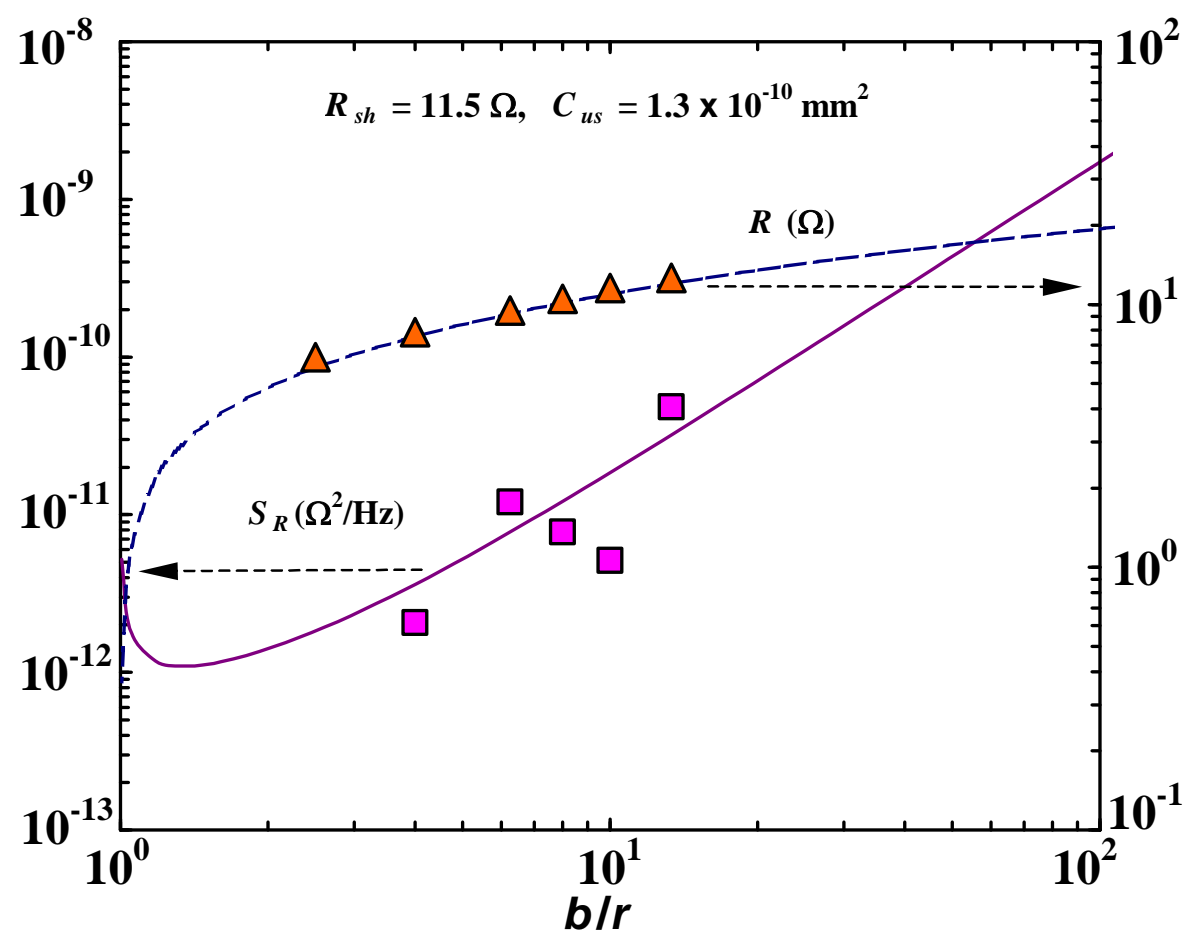

Fig. 5. 
Table 1.

Perfect constriction dominated contacts Interface dominated (poor) contacts, concentric circular equipotentials $(b / r>1)$

$$
\begin{aligned}
& R \quad R_{c}=\frac{R_{s h}}{\pi} \ln \frac{b}{r} \propto \ln \frac{b}{r} \quad \text { (4) } \quad R_{i}=2 \frac{\rho_{i} t_{i}}{\pi r^{2}}=2 \frac{\rho_{\text {cont }}}{\pi r^{2}} \propto \frac{1}{r^{2}} \\
& S_{R} \quad S_{R_{c}}=\frac{R_{s h}{ }^{2} C_{u s}}{f(2 \pi)^{3}}\left[\frac{1}{r^{2}}-\frac{1}{b^{2}}\right] \propto \frac{1}{r^{2}} \quad \text { (9) } \quad S_{R_{i}}=\frac{C_{u s_{i}}}{f}\left[\frac{2 \rho_{c o n t}{ }^{2}}{\pi^{3} r^{6}}\right] \propto \frac{1}{r^{6}}
\end{aligned}
$$

Table 2.

\begin{tabular}{lll}
\hline $\begin{array}{l}\text { Diameter } 2 r \\
(\mathrm{~mm})\end{array}$ & $\begin{array}{l}R \\
(\Omega)\end{array}$ & $\begin{array}{l}S_{R} @ 4 \mathrm{~Hz} \\
\left(\Omega^{2} / \mathrm{Hz}\right)\end{array}$ \\
\hline 1.5 & 12.6 & $4.84 \times 10^{-11}$ \\
2.0 & 11.7 & $5.14 \times 10^{-12}$ \\
2.5 & 10.4 & $7.75 \times 10^{-12}$ \\
3.2 & 09.5 & $1.20 \times 10^{-11}$ \\
5.0 & 07.9 & $2.07 \times 10^{-12}$ \\
8.0 & 06.7 & --- \\
\hline
\end{tabular}

